\title{
Handedness in rats: Blockade of reaching behavior by unilateral 6-OHDA injections into substantia nigra and caudate nucleus
}

\author{
BERT SIEGFRIED and JAN BURES \\ Institute of Physiology, Czechoslovak Academy of Sciences, Prague, Czechoslovakia
}

\begin{abstract}
Unilateral 6-hydroxydopamine (6-OHDA) injections into the nigrostriatal system of rats were used to interfere with a lateralized reaching-for-food habit, the so-called "handedness." Experiment 1: Although injections of 6-OHDA into the substantia nigra $(8 \mu \mathrm{g} / 4 \mu 1)$ ipsilateral $(\mathrm{n}=7)$ to the preferred forelimb had no effect on the overlearned instrumental reaction, reaching was blocked 15 min after contralateral $(n=12)$ injection of the neurotoxin and remained suppressed for the 8 days of testing. At the same time, retrieval of food with the nonpreferred forepaw significantly increased. Although the rats failed to reach into the feeder, behaviors like grasping, grooming, or holding food pellets were intact in the "neglected" forepaw. Nonspecific effects such as changes in activity level or motivation could be ruled out by the ipsilateral-contralateral test paradigm used. The early behavioral asymmetries (blockade of handedness, flexion of the forelimb, and ipsilateral turning) are interpreted as due to interference of 6-OHDA with membrane structures of dopaminergic neurons. Experiment 2: Injections of 6-OHDA into the caudate nucleus contralateral $(30 \mu \mathrm{g} / 5 \mu \mathrm{l}, \mathrm{n}=9)$ to the performing forelimb initially blocked handedness similarly as in Experiment 1 . However, in contrast to nigral injections, reaching recovered within 6 days. Experiment 3: One month after a unilateral 6-OHDA lesion of substantia nigra, naive rats $(n=7)$ acquired the reaching-for-food habit with the paw ipsilateral to the lesion. It is concluded that the blockade of handedness produced by unilateral 6-OHDA lesions of substantia nigra is not due to paralysis of the forelimb but to faulty integration of the contralateral somatosensory signals with contralateral motor patterns.
\end{abstract}

Lateralized 6-hydroxydopamine (6-OHDA) lesions of the nigrostriatal bundle represent an important tool of research into the dopaminergic brain mechanisms. Although the turning tests (Ungerstedt \& Arbuthnott, 1970) and sensory neglect experiments (Ljungberg \& Ungerstedt, 1976; Marshall, Richardson, \& Teitelbaum, 1974) reveal the motor and perceptual manifestations of the resulting brain asymmetry, the mutual relationship of these symptoms remains obscure.

The lesion may induce a global left-right reactivity gradient or cause restricted unilateral deficits. It may also differentially influence automatic innate behavior and voluntary skilled movements. The relative significance of the above alternatives can be estimated by investigation of discrete lateralized instrumental reactions, the acquisition and performance of which require precise sensorimotor integration in well-determined input and output circuits. An example of such reaction is the so-called "handedness"

Bert Siegfried is a postdoctoral fellow of the Swiss National Science Foundation from the Institute of Pharmacology, University of Zurich, Zurich, Switzerland.
(Peterson, 1934), the capability of rats to reach for food into a narrow tube with one forepaw. This acquired skilled movement combines lateralized somesthetic and proprioceptive inputs from the preferred forepaw with complex motor programs integrating the discrete forepaw movement with gross postural adjustments. Results of lesion studies and electrophysiological analysis show that motor cortex, cerebellar hemispheres and basal ganglia participate in the reaching reaction (Castro, 1972a; Dolbakyan, Hernandez-Mesa, \& Bureš, 1977; Hernandez-Mesa \& Bureš, 1977; Islam \& Bureš, 1975; Megirian, Burešová, Bureš, \& Dimond, 1974; Peterson \& Devine, 1963). It is conceivable that basal ganglia not only contribute to elaboration of the reaching movement and to integration of sensory and motor signals, but that they also act as a mediating link between the hypothalamic motivational circuits and the cortical mechanism of the voluntary movement.

The purpose of the present study was to examine the effects of unilateral interference with the nigrostriatal system on the lateralized reaching-for-food habit. The data were presented in a preliminary version earlier (Siegfried \& Bureš, Note 1). 


\section{GENERAL METHOD}

\section{Subjects}

The experiments were performed in 39 male hooded rats (Druckrey strain) aged 2-3 months. The animals were reduced to $80 \%$ of initial body weight and maintained on a 24-h fooddeprivation schedule with water freely available in the home cages.

\section{Apparatus}

The handedness was tested in a plastic chamber $(30 \times 18 \times 15 \mathrm{~cm})$ with a circular opening in the center of the front wall, $5 \mathrm{~cm}$ above the floor. A perspex feeder, a tube $4.5 \mathrm{~cm}$ long with a $1.1-\mathrm{cm}$ internal diameter was attached to the wall opening from outside. A hole, $8 \mathrm{~mm}$ in diameter, in the upper part of the tube made it possible to introduce into the feeder small pellets $(30-50 \mathrm{mg}$ each) prepared from Larsen's wet rat chow. The distance of the food from the feeder was controlled by an adjustable piston. The apparatus is described in detail elsewhere (Hernandez-Mesa \& Bureš, 1977).

\section{Procedure}

Food-deprived rats were introduced into the apparatus and offered food placed close to the feeder entrance. The pellets were gradually moved into the feeding tube until the animal could reach them only with one forepaw. Usually, rats preferred to perform this movement with the left or right forepaw and the preference stabilized after 2-3 days of training. Thirty pellets were given per day and training continued until the animals were able to get pellets placed 2-3 cm deep into the feeder. The rats were allowed to eat freely for $15 \mathrm{~min}$ after they were removed from the testing box. During the testing sessions, food offered in the experimental chamber was limited to 20 pellets per day if not otherwise stated.

\section{Data Analysis}

All trials were divided into three categories, that is, the rat removes the pellet from the tube within $30 \mathrm{sec}$ (1) with the preferred forelimb $(\mathrm{P})$, or (2) with the nonpreferred forelimb $(\mathrm{N})$, or (3) fails to retrieve the pellet during $30 \mathrm{sec}$ after loading the feeder $(\mathrm{O})$. It was also noted whether the preferred or nonpreferred forelimb was assisted by movements of the other paw.

\section{Statistics}

Proportions were either compared with the z-test (Bruning \& Kintz, 1968) or using tables of binomial probability distribution (Clopper \& Pearson, 1934).

\section{Turning Test}

The extent of dopamine neuron degeneration can be estimated in a rotometer test. The dopamine receptors in the denervated striatum are considered to develop a supersensitivity and agents with direct receptor activity, such as apomorphine, cause circling away from the denervated side by preferential activation of the supersensitive receptors (Ungerstedt, 1971a). At the end of Experiments 1 and 2 (9 to 11 days after the 6-ODHA lesion), each rat was placed in a hemispherically shaped bowl $(40 \mathrm{~cm}$ in diameter) and connected by a thin steel wire to a mechanical counter which counted the net difference between completed left or right revolutions. The number of contralateral turns during a 30 -min period, beginning immediately after injection of apomorphine hydrochloride $(.5 \mathrm{mg} / \mathrm{kg}$, calculated as the base, i.p.) was used as an index of the degree of dopamine neuron degeneration.

\section{Histology}

Verification of the location of the cannula tip was limited to a sample of five animals, since dopamine neuron degeneration was checked functionally in a rotometer test. The amount of 6-OHDA used $(8 \mu \mathrm{g} / 4 \mu \mathrm{l})$ guaranteed a large destruction of the nigrostriatal pathway and might have even caused nonspecific damage of brain tissue (Agid, Javoy, Glowinsky, Bouvet, \& Sotelo, 1973).

\section{EXPERIMENT 1}

The first series of experiments compares the effect of unilateral 6-OHDA injection into substantia nigra contralateral or ipsilateral to the preferred forepaw on the overlearned reaching habit and on innate unconditioned motor reactions.

\section{Method}

Well-trained rats $(n=19)$ were anesthetized with Nembutal $(40 \mathrm{mg} / \mathrm{kg}$ ) and a stainless steel guide cannula (gauge $22: .71 \mathrm{~mm}$ outer diameter, . $.41 \mathrm{~mm}$ internal diameter, $16 \mathrm{~mm}$ long) was lowered to a point $1 \mathrm{~mm}$ above the rostral part of the substantia nigra contralateral $(n=12)$ or ipsilateral $(n=7)$ to the preferred forelimb using the following coordinates according to the atlas of Hurt, Hanaway, and Netsky (1971): $1.8 \mathrm{~mm}$ lateral to the superior sagittal sinus, $3.5 \mathrm{~mm}$ anterior, and $3.0 \mathrm{~mm}$ dorsal to the interaural line. (The bar for the upper incisors was $2.5 \mathrm{~mm}$ below the horizontal plane of the interaural line.) Anchoring bolts were placed into slit-like openings in the frontal and occipital bone, and the whole implant was fixed to the skull with acrylate. Two days after surgery, the rats were retrained during 2 days ( 20 pellets/day) in the experimental apparatus. On Day 6, unanesthetized, handrestrained rats were injected during 4 min with $4 \mu \mathrm{l}$ vehicle solution $(.1 \%$ ascorbic acid $(w / v)$ in isotonic saline) into the substantia nigra, using a hand-operated microsyringe. The injection needle (gauge $28: .36 \mathrm{~mm}$ outer diameter, $.15 \mathrm{~mm}$ internal diameter) protruded $1 \mathrm{~mm}$ beyond the tip of the cannula. Reaching was tested during two sessions (0-30 min, 30-60 min, 5 pellets/session) following the control injection. Two hours later, $8 \mu \mathrm{g}$ (base) 6-hydroxydopamine-HCl dissolved in $4 \mu \mathrm{l}$ vehicle solution were applied during $4 \mathrm{~min}$ into the substantia nigra of the same animals. Reaching tests were performed four times on the lesioning day (0-15 min, 15-60 min, 1-2 h, 4-6 h; 5 pellets/session) and once per day ( 20 pellets/day) during the following 8 days.

The grasping reflex and the animal's capability to hold a small food morsel with the affected forelimb (contralateral to the 6-OHDA lesion) were tested in a sample of animals during the postlesion period.

\section{Results}

Figure 1 summarizes the changes of reaching after unilateral injection of $8 \mu \mathrm{g} \mathrm{6-OHDA}$ into the substantia nigra of unanesthetized hand-restrained rats. Intranigral injection of vehicle or 6-OHDA solution ipsilateral to the performing forelimb, and vehicle injection into the contralateral substantia nigra had no effect on reaching: all rats continued to reach with their trained forelimb. Contralateral injection of 6-OHDA had small but significant effect during the first $15 \mathrm{~min}(\mathrm{p}<.01$, binomial probability test), but afterwards reaching with the originally preferred forelimb (P) was almost completely blocked, for the 8 days of testing. Performance with the preferred forelimb further decreased during the 15-60-min test session $(z=6.29, p<.001)$ while reaching trials with the nonpreferred forepaw $(\mathrm{N})$ or trials in which the rats stopped performing or continued unsuccessful attempts (O) significantly increased $(\mathrm{N}: \mathrm{z}=3.10$, $\mathrm{p}<.002 ; \mathrm{O}: \mathrm{z}=4.54, \mathrm{p}<.001)$. The performance deficit was not due to reduced activity or motivation. 

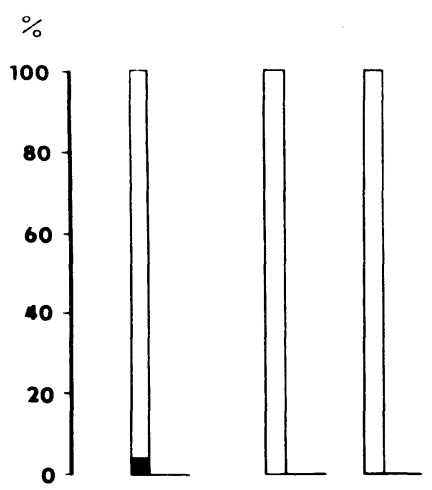

$\%$

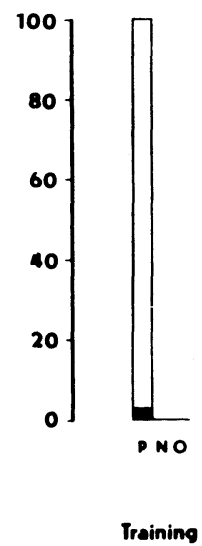

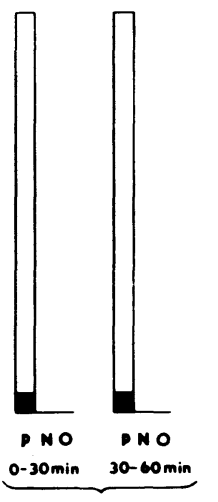

Vehicle
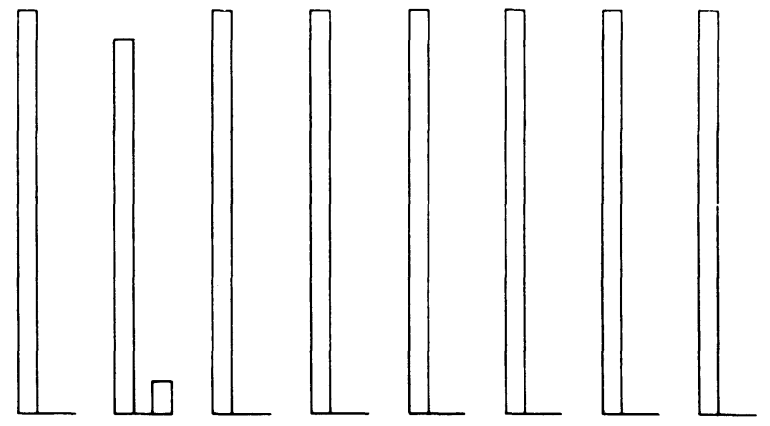

CONTRALATERAL

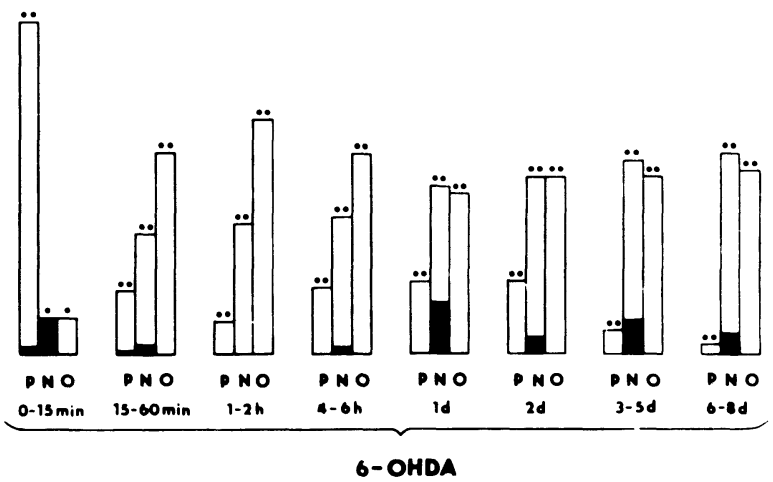

Figure 1. Effect of vehicle and 6-OHDA $(8 \mu \mathrm{g} / 4 \mu \mathrm{l})$ injections into the substantia nigra ipsilateral $(\mathrm{n}=7$ rats $)$ or contralateral ( $n=12$ rats) to the performing forelimb on reaching behavior. The ordinate gives the number of trials (in percentages of the total trials/session) in which the rats used the preferred forelimb (P), the nonpreferred fore$\operatorname{limb}(\mathrm{N})$, or failed to reach $(\mathbf{O})$. Training: Trials after cannula implantation during 2 days preceding the 6-OHDA injection. The black area in the white columns gives the incidence of trials in which the preferred or nonpreferred forelimb was assisted by movements of the other forepaw. Black dots above the columns: one dot: $p<.05$, two dots: $p<.01$, when compared to vehicle sessions (binomial probability test).

Animals with the ipsilateral lesions performed normally, and some rats with contralateral 6-OHDA injection continued to reach with the nonpreferred paw (N) $(8.4 \%$ to $50 \%$ of all trials). Even when the rats were unable to get the pellet $(\mathrm{O})$, they continued unsuccessful reaching attempts and avidly ate food placed on the floor of the testing chamber.

General behavior. The onset of reaching impairment (15-60 min session) coincided in many instances with other behavioral and postural deficits in the forelimb contralateral to the nigral 6-OHDA injection, for example, with the impairment of visual (see Figure 2A) and tactile placing reactions. The most reliable short latency effect of 6-OHDA was a peculiar flexion of the contralateral forelimb, bent at an angle of about $90^{\circ}$ in the wrist (see Figure 2A, left forelimb). Like the reaching impairment, the described deficits lasted for the whole testing period (up to 8 days). The lesioned rats were still able to hold a mor- sel of food with their 6-OHDA affected forelimb (Figure 2B, left forelimb), however, and the grasping reflex was intact in the paw contralateral to the 6-OHDA treated substantia nigra (Figure 2C, left forelimb). During face grooming, the neglected contralateral forelimb was used in concert with the intact one so efficiently that the unilaterally lesioned rats were indistinguishable from nonlesioned rats.

Turning behavior. Spontaneous turning to the side of 6-OHDA injection was observed immediately after injection in 12 rats ( 8 rats injected contralateral and 4 rats injected ipsilateral to the performing limb). In some cases, moderate ipsilateral turning was still present at a time when reaching started to be impaired. The animal would then usually do one turn, attend the Plexiglas tube, try to remove the pellet during 5 to $10 \mathrm{sec}$, and do another ipsilateral turn. During the apomorphine test, the 7 rats with the 6-OHDA lesion ipsilateral to the preferred forelimb 

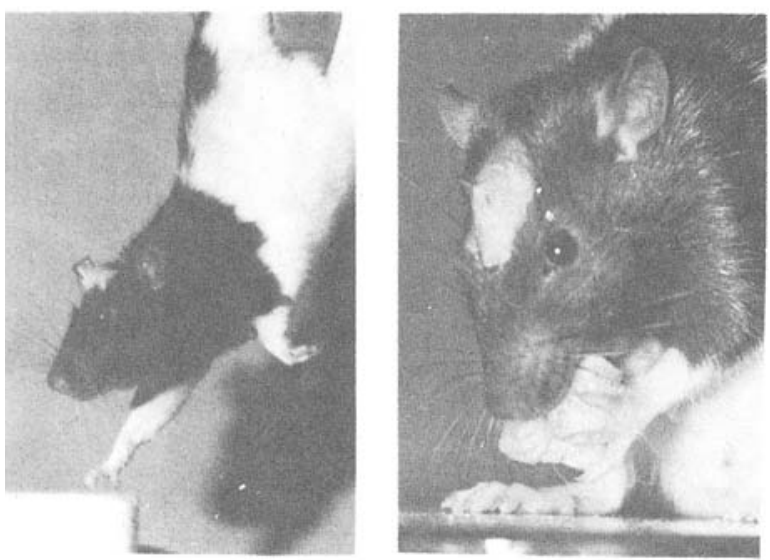

c

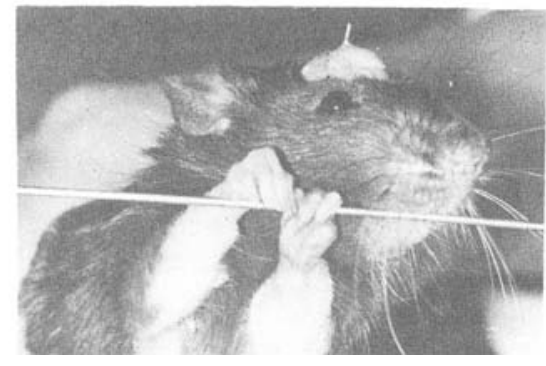

Figure 2. Behavioral tests in rats with a unilateral (right side) 6-OHDA lesion of the substantia nigra. Above left: visual placing reaction: note the failure of placing with the left forelimb and its peculiar flexion. Above right: rat holding a morsel of food with the "neglected" left hand, contralateral to the lesion. In this rat, the right forelimb is denervated. The right hand is therefore not able to hold the food. During eating, however, it provides counterpressure to the neglected limb. Below: rat correctly grasping with the "neglected" left hand. Again, the right forelimb is denervated and the grasping reflex with this hand is absent.

scored $18.0 \pm 8.7$ (SEM) contralateral turns per $30 \mathrm{~min}$. Apomorphine turning was significantly stronger in animals with contralateral 6-OHDA lesion: the 12 rats scored $76.2 \pm 25.0$ contralateral turns per $30 \min (d f=17, t=2.20, p<.05)$. Reaching was also impaired in the 3 out of 12 contralaterally lesioned rats which showed no turning after apomorphine.

Histology. The tip of the injection needle was positioned in substantia nigra pars reticulata. Necrotic lesions had a diameter of 1.2 to $1.5 \mathrm{~mm}$. The minimum and maximum area of necrotic tissue was similar as shown in an earlier report (Siegfried \& Bureš, 1979) and corresponded to the findings of other authors (Agrid et al., 1973) using the same volume and concentration of 6-OHDA.

\section{Discussion}

6-OHDA injections into the substantia nigra, contralateral but not ipsilateral to the performing forelimb, blocked reaching within the first hour. Reach- ing did not recover during the 8-day testing period, despite daily training sessions. The maintained responding during the first $15 \mathrm{~min}$ after injection corresponds to the diffusion of 6-OHDA. The subsequent short latency impairment of reaching cannot be due to degeneration but rather to a functional blockade of the nigrostriatal pathway. This is indicated by the ipsilateral turning recorded immediately after injection and concurs with the increased retention of striatally injected ${ }^{3} \mathrm{H}$-dopamine found after such a lesion (Ungerstedt, 1971b). Biochemical and electrophysiological data show that peripheral application of 6-OHDA produces within $1 \mathrm{~h}$ damage of the membrane of the adrenergic nerve terminals (Haeusler, 1971; Jonsson \& Sachs, 1972), manifested by a loss of conductivity, increase of calcium permeability, and damage of the noradrenaline uptake mechanism. The 6-OHDA-induced blockade of $\alpha$-adrenergic receptors (Haeusler, 1971; Nakamura \& Thoenen, 1971) might be significant in light of the recently developed concept of "autoreceptors" (Carlsson, 1975), receptors distributed over the surface of a neuron, which exhibit sensitivity to the transmitter released by the neuron. The early behavioral asymmetries (blockade of handedness, flexion of the forelimb, and ipsilateral turning) seen after unilateral nigral 6-OHDA injections are thus likely to be due to interference of 6-OHDA with membrane structures of dopaminergic neurons and axons, which in turn decrease impulse activity of the nigrostriatal system on the treated side. Gradual transition of the early 6-OHDA effects into degeneration of the nigrostriatal pathway prevents behavioral separation of the early and late consequences of nigral injection.

6-OHDA injections into substantia nigra impaired the acquired reaching movement, but did not interfere with automatic movements of the affected forelimb, performed in concert with the intact limb (grooming, food holding) and with simple reflexes (grasping). This finding is in accordance with previous reports on nigral 6-OHDA lesions (Ljungberg \& Ungerstedt, 1976; Marshall et al., 1974), and also with the preservation of spontaneous grooming in decerebrated rats (Grill \& Norgren, 1978).

Whereas a previous study, in which colchicine injections into substantia nigra reversibly blocked handedness behavior (Siegfried \& Bureš, Note 1), revealed importance of nigral cells for this habit, the present data suggest specific involvement of ascending dopaminergic neurons. The possibility must be taken into account, however, that nonspecific destruction of nigral nondopaminergic cells (Di Chiara, Olianas, Del Fiacco, Spano, \& Tagliamonte, 1977; Fibiger, Pudritz, McGeer, \& McGeer, 1972; Schwartz, Gunn, Sharp, \& Evarts, 1976) as well as of structures outside the substantia nigra (partial damage of the peduncles, red nucleus) also contributed to the handedness failure. 


\section{EXPERIMENT 2}

The rather large dose and volume of 6-OHDA injected into substantia nigra in Experiment 1 could have affected not only the ascending nigrostriatal bundle but also efferent pathways at the nigral level (Fibiger et al., 1972) or, particularly, motor pathways running close to substantia nigra such as corticospinal and rubrospinal tracts. Involvement of the dopaminergic neurons in handedness can be demonstrated more convincingly by interference at the neostriatal level. The purpose of Experiment 2 was to investigate the effects of 6-OHDA injections into the caudate nucleus.

\section{Method}

Surgery and testing procedure were the same as described in Experiment 1, except that, on Day 6, the vehicle $(5 \mu \mathrm{l})$ and, $2 \mathrm{~h}$ later, the drug solution $(30 \mu \mathrm{g}$ 6-OHDA in $5 \mu \mathrm{l}$ vehicle) were injected for $5 \mathrm{~min}$ into the caudate nucleus contralateral to the performing forelimb $(n=9)$, according to the stereotaxic atlas by Fifková and Maršala (1967): AP: -1.0 , L: 2.5, V: 6.0 (AP 0.0 corresponds to bregma; the horizontal plane passes through bregma and a point $1 \mathrm{~mm}$ above lambda.) Reaching behavior was tested during several sessions on Day 6 (5 pellets/session) and once per day ( 20 pellets) during the following 8 days.

\section{Results}

The 6-OHDA injection into the striatum contralateral to the performing forelimb affected reaching similarly as intranigral 6-OHDA injection. After ve- hicle injection and during the first $15 \mathrm{~min}$ after 6-OHDA injection, rats continued to use their preferred forelimb. Reaching was significantly impaired 15 to 60 min after injection $(\mathrm{p}<.01$, when compared to vehicle sessions, binomial probability test; $z=5.17$, $\mathrm{p}<.001$, when compared to the $0-15 \mathrm{~min}$ session; Figure 3), and remained low during 2 postinjection days. Consequently, the number of trials in which the rats failed to reach the pellet $(\mathrm{O})$ significantly increased $(\mathrm{p}<.01$, when compared to vehicle sessions, binomial probability test; $\mathrm{z}=4.40, \mathrm{p}<.001$, when compared to the $0-15$-min session). In most cases (70\%-80\% per session), rats completely stopped performing, although they still continued to eat food pellets placed in the testing chamber. This contrasts with the results after intranigral injection in which most trials of the $\mathrm{O}$-group consisted of unsuccessful reaching. The nonpreferred forepaw $(\mathrm{N})$ was used less frequently after intrastriatal $(6.7 \%$ to $10 \%)$ than after intranigral $(10 \%$ to $41.6 \%)$ injections on the 2 postinjection days.

The most important difference between the two lesions was significant recovery of reaching with the preferred forelimb starting 3 days after 6-OHDA injection into the contralateral caudate nucleus. Performance significantly increased from Day 2 to Day 3 $(\mathrm{z}=2.40, \mathrm{p}<.02)$ but remained significantly lower than in the vehicle trials $(\mathrm{p}<.01)$. As a consequence, trials in which rats failed reaching $(\mathrm{O})$ significantly decreased during this period $(\mathrm{z}=2.22, \mathrm{p}<.03)$. Reaching with the preferred forelimb further recovered during the 6- to 8-day postinjection period.
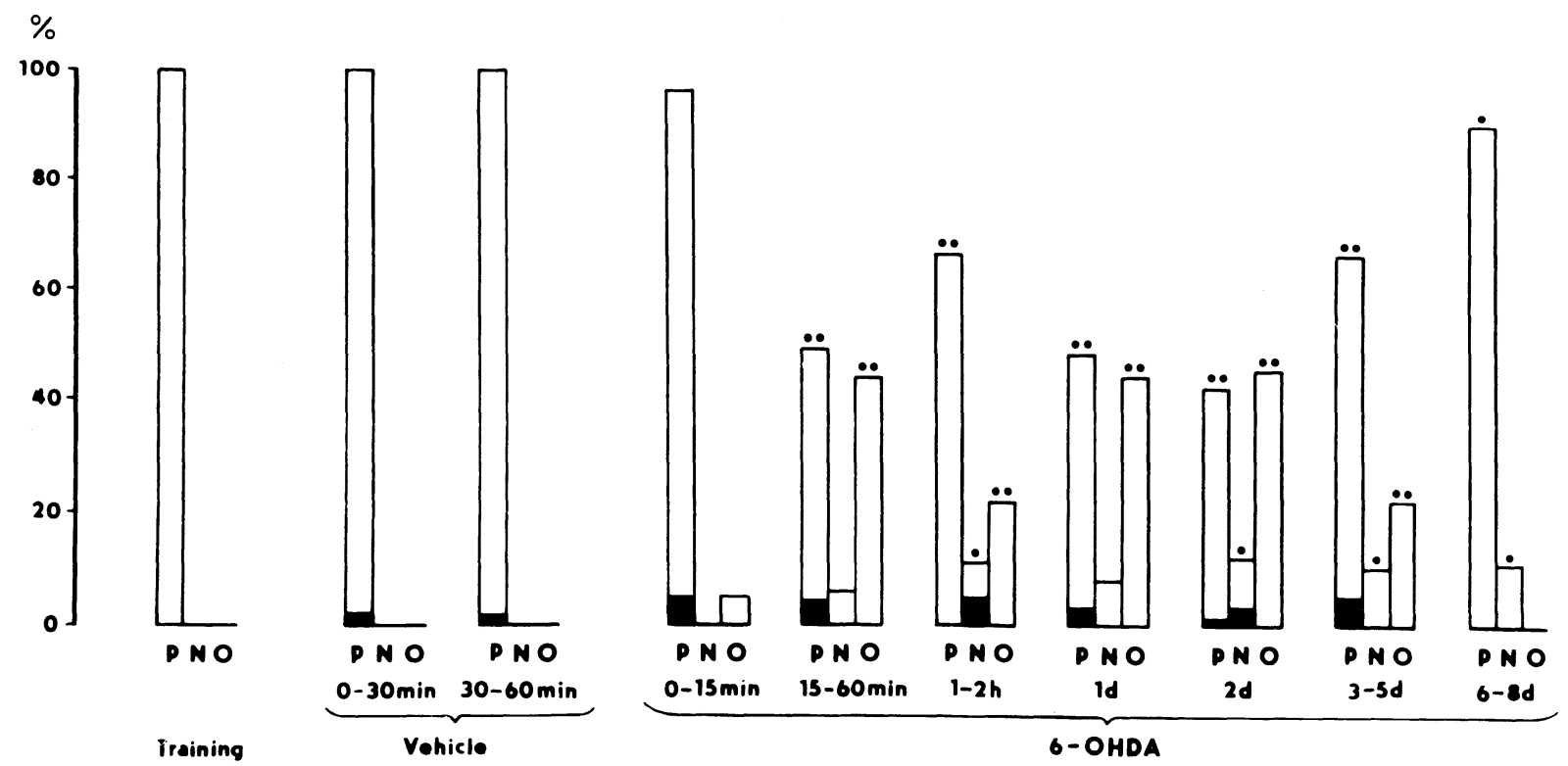

Figure 3. Effect of vehicle and 6-OHDA $(30 \mu \mathrm{g} / 5 \mu \mathrm{l})$ injection into the caudate nucleus contralateral $(\mathrm{n}=9$ rats) to the performing limb on reaching behavior. For further details, see legend of Figure 1. 
General behavior. The typical flexion of the forelimb was not as clearly expressed as after intranigral 6-OHDA injections.

Turning behavior. Whereas apomorphine induced contralateral turning in five of nine lesioned rats [55.2 \pm 27.8 (SEM) turns/30 $\mathrm{min}, \mathrm{n}=5$, range: $14-164$ turns], two rats turned ipsilateral to the lesion (14 and 18 turns $/ 30 \mathrm{~min}$ ) and two other rats showed no turning tendency after apomorphine. No correlation was found between the intensity of turning and the reaching impairment.

Histology. The tips of the striatal cannulae were positioned 1.5 to $2.0 \mathrm{~mm}$ above the commissura anterior. The necrotic lesions were of spherical and/or ovoidal shape with a diameter of 1.2 to $1.5 \mathrm{~mm}$.

\section{Discussion}

The initial effects of striatal 6-OHDA injection are the same as those of intranigral injection, but gradual recovery of reaching shows that the damage can be compensated by the remnants of neostriatal tissue. The less frequent transfer to the nonpreferred forepaw indicates that the compensatory processes concentrated on restitution of the original habit rather than on formation of a new one. Complete absence of responding encountered in $30 \%$ to $35 \%$ of trials was not due to general interference with consummatory behavior, since all rats readily ate food placed into the test chamber, but may reflect weakening of the motivational input into the neural circuits of reaching. Finally, intensity and direction of apomorphine-induced turning appear to depend on the extent of striatal damage. The animals turned contralaterally when there were enough of hypersensitive dopaminergic receptors left in the damaged neostriatum and ipsilaterally when the loss of striatal tissue was too severe (Glick, Jerussi, \& Fleisher, 1976).

\section{EXPERIMENT 3}

In Experiments 1 and 2, reaching was impaired by lateralized 6-OHDA injection applied to the hemisphere contralateral to the naturally preferred forepaw. The possibility must be taken into account that handedness expresses innate asymmetry of the nigrostriatal system (Glick, Jerussi, \& Zimmerberg, 1977), which is further increased by training of the lateralized habit. Such a conclusion is supported by the difference in the intensity of apomorphine turning after 6-OHDA injection into hemispheres controlling the preferred and nonpreferred forelimbs, obtained in Experiment 1. In order to test this hypothesis, asymmetry of the nigrostriatal system was induced by 6-OHDA injection to naive animals, the handedness of which was determined after full development of the lesion.

\section{Method}

Eleven rats were implanted with a cannula positioned $1 \mathrm{~mm}$ above the right substantia nigra, as described in Experiment 1. Two days after surgery, they were lesioned with 6-OHDA $(8 \mu \mathrm{g} / 4 \mu \mathrm{l})$. One week before handedness training began, on postlesion Day 25, they were put on a 24-h food deprivation schedule with water freely available. Previous to the 30 pellets of the first training session, rats were offered 10 pellets placed .5 to $1.5 \mathrm{~cm}$ deep into the feeder. The daily training session was stopped after 30 pellets were successfully removed from the tube or after 30 min maximally. Training lasted for 5 days. One hundred thirty rats used in preliminary handedness testing served as the intact control group. The deprivation conditions and training procedure were the same as in the experimental animals.

\section{Results}

Out of 130 control rats, 85 showed a clear left or right forepaw preference 3 days after training, and the number of right-handed $(n=38)$ and left-handed $(n=47)$ rats was similar. Out of 11 rats with 6-OHDA lesions of the right substantia nigra, 7 acquired the reaching habit, all of them with the forelimb ipsilateral (right) to the lesion (Figure 4). The percentage of trials in which one forelimb was assisted by the other (Figure 4, black columns) was similar in the control and lesioned rats and decreased similarly in both groups from the first to the third testing day. Furthermore, the proportion of learners in the lesioned group $(63.6 \%)$ was similar to normal rats $(65 \%)$.

Turning behavior. Apomorphine-induced contralateral turning occurred in three of the seven 6-OHDA lesioned rats that learned the reaching habit $(54.0 \pm 4.7$ turns $/ 30 \mathrm{~min}$ ), and in one of the nonlearner rats (65 turns/30 min).

\section{Discussion}

Unilateral 6-OHDA lesions of substantia nigra did not cause a general deficit of learning: the lesioned rats were indistinguishable from the control group in the acquisition of the habit. The preferential use of the right forepaw supports the notion that handedness and other instances of hemispheric dominance can be due to nigrostriatal asymmetry (Glick et al., 1977). Although some of the initial symptoms of the nigral damage were compensated at the time of testing, inadequate control of the contralateral "neglected" forepaw discouraged its use in the first attempts to reach into the feeder and favored the use of the ipsilateral forepaw. The possibility of influencing handedness by a much weaker functional bias (a few forced reachings performed with one forepaw) was described by Wentworth (1933) and by Burešová, Bureš, and Beran (1958). 

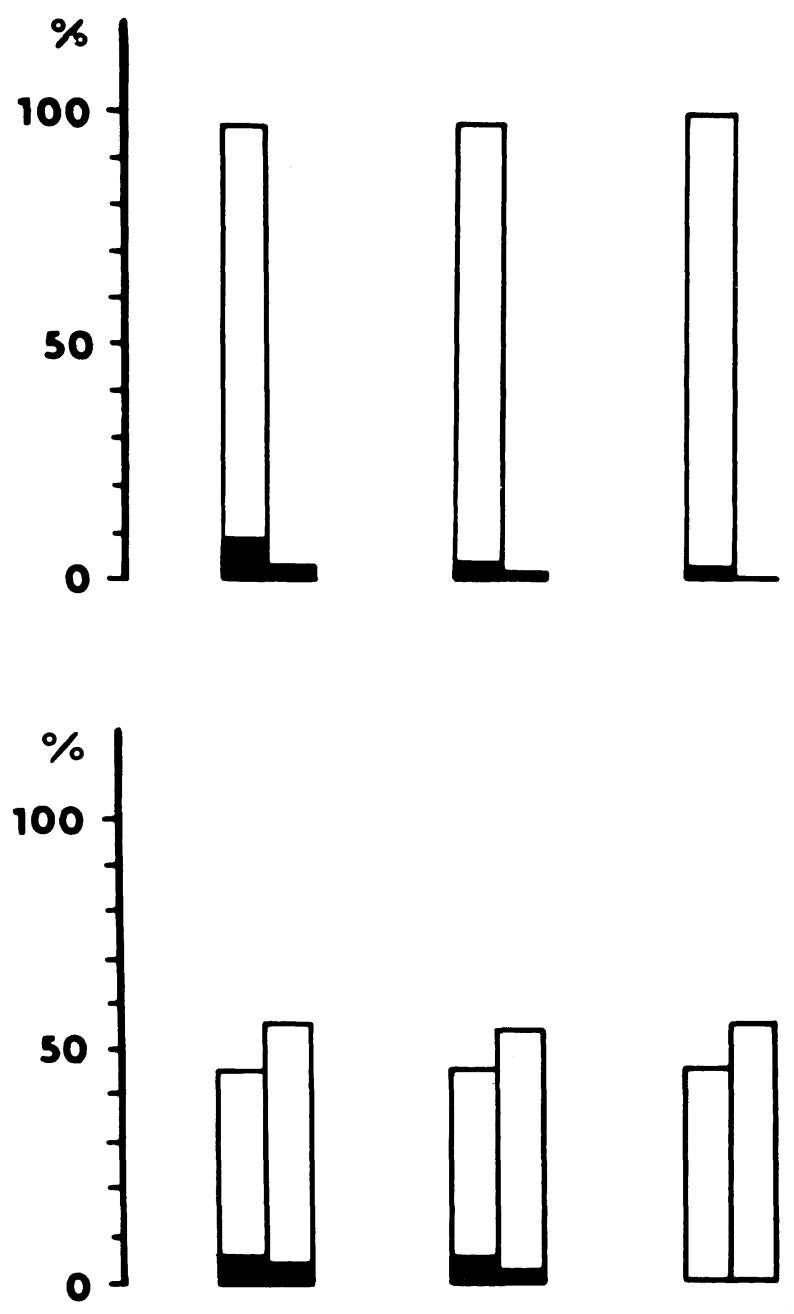

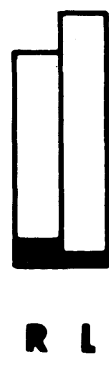

2

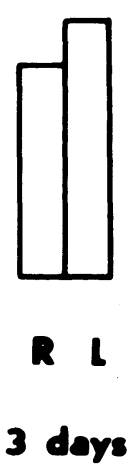

Figure 4. Number of trials (in percentages of all reaching trials) with the right (R) or left (L) forepaw during the first three daily handedness-training sessions in seven rats with 6-OHDA lesion of the right substantia nigra (above) and in control rats $(n=85$, below) Other descriptions as in Figure 1.

\section{GENERAL DISCUSSION}

Impairment of reaching by lesions or pharmacological blockade of contralateral substantia nigra or caudate nucleus can be due to interference with motor control or with sensorimotor integration. The present experiments show that it is not due to paralysis of the forepaw but to the loss of its purposeful control. The data contribute to the characterization of the neglect syndrome, produced by unilateral 6-OHDA lesions (Ljungberg \& Ungerstedt, 1976;
Marshall et al., 1974; Siegfried \& Bureš, 1978, 1979), that is, blockade of handedness is obviously due to faulty integration of the contralateral somatosensory signals with the contralateral motor patterns. Nonspecific effects, such as changes in activity level or motivation to eat, could be ruled out by the ipsilateralcontralateral test paradigm used. The combination of lateralized behaviors with unilateral 6-OHDA lesions was recently used to show dependence of striatal (Phillips, Carter, \& Fibiger, 1976) and lateral hypothalamic (Koob, Fray, \& Iversen, 1978) self-stimulation and cortical spreading depression-induced feeding (Siegfried, Hefti, Lichtensteiger, \& Huston, 1979) on the nigrostriatal dopamine system.

Comparable results were obtained by Turner (1973) and by Hoyman, Weese, and Frommer (Note 2), who reported that rats with unilateral lesions of lateral hypothalamus are unable to learn operant head movements to the side contralateral to the lesion. In accordance with this view is the work by Hansing, Schwartzbaum, and Thompson (1968) and by Levine, Ferguson, Kreinick, Gustavson, and Schwartzbaum (1971), who showed that a unilateral lesion of caudate nucleus or globus pallidus prevented lever pressing with the contralateral forepaw. The impairment was characterized as a loss of the capacity to utilize the affected forelimb in performing organized movements. Evidence also exists that stimulatory effects at the level of substantia nigra or caudate nucleus interfere with forelimb performance, that is, movement-synchronized stimulation of the caudate nucleus prevented the completion of the reach (Hernandez-Mesa \& Bureš, 1977).

Electrophysiological experiments (Dolbakyan et al., 1977; Hernandez-Mesa \& Bureš, 1978) showed reaching-related changes of activity in $50 \%$ of neurons in the contralateral caudate nucleus. Damage, blockade, or stimulation of the striatum, and especially of its nigral dopaminergic input, obviously interferes with the patterned activity of caudate neurons which seems to play an important role in the performance of the habit.

The weak and reversible effect of neostriatal 6-OHDA injection is probably due to the smaller extent of the lesion. Gaddy and Neill (1977) reported after bilateral injection of a comparable amount of 6-OHDA into the neostriatum of rats transient impairment of various behaviors, including locomotor activity, feeding, and self-stimulation. Self-stimulation was most severely suppressed, but recovered to prelesion level after 7 days, that is, with a similar rate as the reaching reaction in Experiment 2. The stronger and irreversible effect of substantia nigra lesions is either due to more severe changes at the striatal level or to additional effects of 6-OHDA injection into substantia nigra on motor function, for example, to partial damage of the pyramidal 
tract at the level of cerebral peduncles. Similar irreversible failure of reaching was observed after pyramidal tract section (Castro, 1972b).

\section{REFERENCE NOTES}

1. Siegfried, B., \& Bureš, J. Comparison of 6-OHDA and colchicine blockade of the nigro-striatal and cortical components of handedness in rats. Paper presented at the E. B. B. S. Workshop Meeting “The Neostriatum," April 16-20, 1978, Vingstedt Centret, Denmark.

2. Hoyman, L., Weese, G. D., \& Frommer, G. P. Tactile discrimination during neglect following unilateral lesions in lateral hypothalamus of rats. Paper presented at Seventh Annual Meeting of the Society for Neuroscience, Anaheim, California, 1977 (Abstract No. 1548).

\section{REFERENCES}

Agid, Y., Javoy, F., Glowinsky, J., Bouvet, D., \& Sotelo, C. Injection of 6-hydroxydopamine into the substantia nigra of the rat. II. Diffusion and specificity. Brain Research, 1973, 58, 291-301.

Bruning, J. L., \& Kintz, B. L. Computational handbook of statistics. Glenview, Ill: Scott, Foresman, 1968.

Bureśová, O., Bureś, J., \& Beran, V. A contribution to the problem of the "dominant" hemisphere in rats. Physiologia Bohemoslovaca, 1958, 7, 29-37.

CARlsson, A. Receptor-mediated control of dopamine metabolism. In E. Usdin \& W. E. Bunney (Eds.), Pre- and postsynaptic receptors. New York: Marcel Dekker, 1975.

CASTro, A. J. The effects of cortical ablations on digital usage in the rat. Brain Research, 1972a, 37, 173-185.

CAstro, A. J. Motor performance in rats. The effects of pyramidal tract section. Brain Research, 1972b, 44, 313-323.

Clopper, J., \& Pearson F. S. The use of confidence or fiducial limits illustrated in the case of binomial. Biometrika, 1934, 26, 404-413.

Di Chiara, G., Olianas, M., Del Fiacco, M., Spano, P. F., \& TAgLiamonte, A. Intranigral kainic acid is evidence that nigral non-dopaminergic neurones control posture. Nature, 1977, 268 , 743-745.

Dolbakyan, E., Hernandez-Mesa, N., \& Burě́, J. Skilled forelimb movements and unit activity in motor cortex and caudate nucleus in rats. Neuroscience, 1977, 2, 73-80.

Fibiger, H. C., Pudritz, R. E., McGeer, P. L., \& McGeer, E. G. Axonal transport in nigrostriatal and nigrothalamic neurons: Effects of medial forebrain bundle lesions and 6-hydroxydopamine. Journal of Neurochemistry, 1972, 19, 1697-1708.

Fifková, E., \& MarŠala, J. Stereotaxic atlases for the cat, rabbit, and rat. In J. Bureš, M. Petrán̆, and J. Zachar (Eds.), Electrophysiological methods in biological research. Prague: Publishing House of the Czechoslovak Academy of Sciences, 1967.

Gaddy, J. R. \& Neill, D. B. Differential behavioral changes following intrastriatal application of 6-hydroxydopamine. Brain Research, 1977, 119, 439-446.

Glick, S. D., Jerussi, T. P., \& Fleisher, L. N. Turning in circles: The neuropharmacalogy of rotation. Life Sciences, 1976, 18, 889-896.

Glick, S. D., Jerussi, T. P., \& ZimmerberG, B. Behavioral and neuropharmacological correlates of nigro-striatal asymmetry in rats. In S. Harnad, R. W. Doty, L. Goldstein, J. Janes, \& G. Krauthamer (Eds.), Lateralisation in the nervous system. New York: Academic Press, 1977.
Grill, H. J., \& Norgren, R. Neurological tests and behavioral deficits in chronic thalamic and chronic decerebrate rats. Brain Research, 1978, 143, 299-312.

Haeusler, G. Early pre- and postjunctional effects of 6-hydroxydopamine. Journal of Pharmacology and Experimental Therapeutics, 1971, 178, 49-62.

Hansing, R. A., Schwartzbaum, J. S., \& Thompson, J. B. Operant behavior following unilateral and bilateral caudate lesions in the rat. Journal of Comparative and Physiological Psychology, 1968, 66, 378-388.

Hernandez-Mesa, N., \& Bureš, J. Impairment of lateralized reaching by movement-synchronized stimulation of motor centers in rats. Experimental Neurology, 1977, 57, 67-80.

Hernandez-Mesa, N., \& Buré̌, J. Skilled forelimb movements and unit activity of cerebellar cortex and dentate nucleus in rats. Physiologia Bohemoslovaca, 1978, 27, 199-208.

Hurt, C. A., Hanaway, J., \& Netsky, M. G. Stereotaxic atlas of the mesencephalon in the albino rat. Confinia Neurologica, 1971, 33, 93-115.

Islam, S., \& Bures, J. Interaction between the activity of an epileptic focus and discrete skilled movements in rats. Electroencephalography and Clinical Neurophysiology, 1975, 39. 651-656.

Jonsson, G., \& Sachs, Ch. Degnerative and nondegenerative effects of 6-hydroxydopamine on adrenergic nerves. Journal of Pharmacology and Experimental Therapeutics, 1972, 180, 625-635.

Kоoв, G. F., Fray, P. J., \& Iversen, S. D. Self-stimulation in the lateral hypothalamus and locus coeruleus after specific unilateral lesions of the dopamine system. Brain Research, 1978, 146, $123-140$

Levine, M. S., Ferguson, N., Kreinick, C. J., Gustavson, J. W., \& Schwartzbaum, J. S. Sensorimotor dysfunctions and aphagia and adipsia following pallidal lesions in rats. Journal of Comparative and Physiological Psychology, 1971, 77, 282-293.

LuUNGBerg, T., \& UngerstedT, U. Sensory inattention produced by 6-hydroxydopamine-induced degeneration of ascending dopamine neurons in the brain. Experimental Neurology, 1976, $53,585-600$.

Marshall, J. F., Richardson, S., \& Teitelbaum, P. Nigrostriatal bundle damage and the lateral hypothalamic syndrome. Journal of Comparative and Physiological Psychology, 1974, 87, 808-830.

Megirian, D., Burešová, O., Bureš, J., \& Dimond, S. Electrophysiological correlates of discrete forelimb movements in rats. Electroencephalography and Clinical Neurophysiology, 1974, 36, 131-139.

Nakamura, K., \& Thoenen, H. Hypothermia induced by intraventricular administration of 6-hydroxydopamine in rats. European Journal of Pharmacology, 1971, 16, 46-53.

Peterson, G. M. Mechanisms of handedness in the rat. Comparative Psychology Monographs, 1934, 9, 1-67.

Peterson, G. M., \& Devine, I. M. Transfer in handedness in the rat resulting from cortical lesions after limited forced practice. Journal of Comparative and Physiological Psychology, $1963,56,752-756$

Phillips, A. G., Carter, D. A., \& Fibiger, H. C. Dopaminergic substrates of intracranial self-stimulation in the caudateputamen, Brain Research, 1976, 104, 222-232.

Schwartz, W. J., Gunn, R. H., Sharp, F. R., \& Evarts, E. V. Unilateral electrolytic lesions of the substantia nigra cause contralateral circling in rats. Brain Research, 1976, 105, 358361 .

Siegfried, B., \& Bureš, J. Asymmetry of EEG arousal in rats with unilateral 6-OHDA lesions of substantia nigra: Quantification of neglect. Experimental Neurology, 1978, 62, 173-190.

Siegrried, B., \& Bureš, J. Conditioning compensates the neglect due to unilateral 6-OHDA lesions of substantia nigra in rats. Brain Research, 1979, 167, 139-155. 
Siegfried, B., Hefti, F., Lichtensteiger, W., \& Huston, J. P. Lateralized hunger: Ipsilateral attenuation of cortical spreading depression-induced feeding after unilateral 6-OHDA injection into the substantia nigra. Brain Research, 1979, 160, 327-340.

Turner, B. H. Sensorimotor syndrome produced by lesions of the amygdala and lateral hypothalamus. Journal of comparative and Physiological Psychology, 1973, 82, 37-47.

Ungerstedt, U. Postsynaptic supersensitivity after 6-hydroxydopamine induced degeneration of the nigrostriatal dopamine system in the rat brain. Acta Physiologica Scandinavica, 1971a, 82, (Suppl. 367), 69-93.

UNGERSTEDT, U. Histochemical studies on the effects of intracerebral and intraventricular injections of 6-hydroxydopamine on monoamine neurons in the rat brain. In T. Malmfors and $\mathrm{H}$. Thoenen (Eds.), 6-hydroxydopamine and catecholamine neurons. Amsterdam: North-Holland, 1971b.

Ungerstedt, U., \& Arbuthnott, G. Quantitative recording of rotational behavior in rats after 6-hydroxy-dopamine lesions of the nigrostriatal dopamine system. Brain Research, 1970, 24, 485-493.

WENTwORTh, K. L. The effect of early reaches on handedness in the rat: A preliminary study. Journal of Genetic Psychology, $1933,52,429-432$.

(Received for publication January 15, 1979; revision accepted July 1, 1979.) 